\title{
Reabilitação Pulmonar com Treinamento de Equilíbrio em Pacientes com Doença Pulmonar Obstrutiva Crônica: Uma Revisão Sistemática
}

Tamires Daros dos Santos, ${ }^{1}$ Sheila Jacques Oppitz, ${ }^{2}$ Yessa do Prado Albuquerque, ${ }^{2}$ Adriane Schmidt Pasqualoto, ${ }^{2}$ Aron Ferreira da Silveira, ${ }^{2}$ Isabella Martins de Albuquerque ${ }^{2}$

\begin{abstract}
RESUMO
O objetivo do estudo foi revisar sistematicamente a literatura sobre a eficácia da adição do treino de equilíbrio (TE) em programas de reabilitação pulmonar (RP) comparado à RP convencional na melhora do equilíbrio postural de indivíduos com Doença Pulmonar Obstrutiva Crônica (DPOC). A estratégia de busca foi realizada nas bases de dados PubMed/Medline, SciELO, Lilacs, PEDro, Scopus e Web of Science, sem restrição quanto ao ano de publicação ou idioma, conforme recomendações do Prisma. Os critérios de inclusão contemplaram: ensaios clínicos envolvendo o TE na RP para melhora do equilíbrio postural em sujeitos com DPOC, publicados até junho de 2019. Dois revisores selecionaram os estudos, extraíram os dados e avaliaram o risco de viés, utilizando o Handbook da Cochrane, de forma independente. Dos 172 estudos potencialmente elegíveis, 2 foram incluídos, compreendendo um total de 107 pacientes com DPOC, dos quais 56 foram alocados no grupo intervenção (TE adicionalmente à RP convencional) e os demais foram inseridos no grupo controle (RP convencional). O TE adicionalmente à RP propiciou melhora no equilíbrio postural em relação à RP convencional, entretanto não foram encontradas diferenças significativas entre os grupos quanto ao nível de confiança na realização de atividades específicas e tolerância ao exercício. Os estudos apresentaram baixo risco de viés (alta qualidade). Sugere-se que a adição do TE em programas de RP convencional seja mais eficaz na melhora do equilíbrio postural em comparação à RP convencional, entretanto, devido ao reduzido número de estudos disponíveis, não há evidência robusta para tomada de decisão clínica.
\end{abstract}

Palavras-chave: Doença pulmonar obstrutiva crônica. Equilíbrio postural. Reabilitação. Revisão.

PROSPECTIVE REGISTER OF SYSTEMATIC REVIEW (PROSPERO): CRD42019128731

PULMONARY REHABILITATION WITH BALANCE TRAINING IN CHRONIC OBSTRUCTIVE PULMONARY DISEASE: A SYSTEMATIC REVIEW

\section{ABSTRACT}

The objective of this study was to systematically review the literature on the efficacy of adding balance training (BT) into pulmonary rehabilitation (PR) programs, in comparison to conventional PR to improve postural balance in individuals with chronic obstructive pulmonary disease (COPD). The search strategy was applied in the PubMed/MEDLINE, SciELO, LILACS, PEDro, Scopus and Web of Science databases, with no restriction as regards the year of publication or language, according to the PRISMA recommendations. Inclusion criteria were: clinical trials involving BT in PR to improve postural balance in subjects with COPD, published up to June 2019. Two reviewers selected the studies, extracted the data and assessed the risk of bias, using Cochrane's Handbook, independently. Of the 172 potentially eligible studies, 2 were included, with a total of 107 patients with COPD, of whom 56 were allocated in the intervention group (BT additionally to conventional PR) and the others in the control group (conventional PR). Balance training additionally to PR provided an improvement in postural balance in relation to conventional PR, however no significant differences were found between the groups regarding the level of confidence in performing specific activities and tolerance to the exercise. The studies presented a low risk of bias (high quality). These findings suggest that the addition of BT into conventional PR programs is more effective to improve postural balance in comparison to conventional PR. However, given the low number of studies available, there is no robust evidence to support clinical decision making.

Keywords: Pulmonary Disease. Chronic obstructive. Postural balance. Rehabilitation. Review.

RECEBIDO EM: 7/8/2019

MODIFICAÇÕES SOLICITADAS EM: 2/5/2020

ACEITO EM: 15/5/2020

\footnotetext{
Universidade Federal de Santa Maria (UFSM) - Santa Maria/RS, Brasil. Avenida Ângelo Bolsson, n 357, Apto. 102, Bairro Duque de Caxias, CEP $97070-000$, Santa Maria, Rio Grande do Sul, Brasil. http://lattes.cnpq.br/2644450723580402. https://orcid.org/0000-0002-4637-4185. tamires.daros@gmail.com.

2 Universidade Federal de Santa Maria (UFSM) - Santa Maria/RS, Brasil.
} 


\section{INTRODUÇÃO}

A Doença Pulmonar Obstrutiva Crônica (DPOC) é uma das principais causas de morbidade e mortalidade, representando um importante problema de saúde pública na atualidade, pois compreende a quarta causa de óbito em âmbito mundial com estimativa de se tornar a terceira até o ano de 2020 (GOLD, 2019). Em 2016 ocorreram aproximadamente $56,9 \mathrm{mi}-$ Ihões de mortes no mundo; dessas, 3 milhões foram decorrentes da DPOC (WHO, 2018).

A principal limitação fisiológica da DPOC é a piora da função pulmonar, porém com a progressão da doença diversas alterações sistêmicas ocorrem (ROCHA et al., 2017). Entre essas, crescente número de evidências tem demonstrado importante déficit no controle do equilíbrio postural de sujeitos com DPOC quando comparado aos saudáveis (PORTO et al., 2017) e, consequentemente, um risco aumentado de quedas (PORTO et al., 2018). Tal premissa é corroborada por estudo que demonstrou maior prevalência de quedas nessa população (44\% a 51\%) comparado aos sujeitos saudáveis (29\% a 33\%) (BEAUCHAMP et al., 2012). Cabe destacar que o equilíbrio postural compreende a capacidade de manter o centro de massa dentro da base de suporte, sendo resultado da integração complexa neuromuscular, somatossensorial e do sistema nervoso central subjacente (SHUMWAY-COOK; WOOLLACOTT, 2007).

Adicionalmente, a osteoporose é altamente prevalente e frequentemente negligenciada na DPOC, muitas vezes sendo diagnosticada apenas após uma fratura (GRAUMAM et al., 2018). Considerando os mecanismos responsáveis pela osteoporose nestes pacientes, a inflamação sistêmica contribui para a perda de peso e massa magra (GRAAT-VERBOOM et al., 2011), o uso de glicocorticoides diminui a densidade óssea e aumenta o risco de fraturas (MOON; SIN, 2019), a inatividade física favorece a redução da densidade mineral óssea (LIU et al., 2015) e níveis reduzidos de vitamina D culminam em menor mineralização, reduzindo a força óssea (KOKTURK et al., 2018). Partindo dessa premissa, a ocorrência de fratura como consequência da osteoporose pode favorecer a incapacidade e mortalidade, dessa forma as alterações no equilíbrio são ainda mais preocupantes nesta população (GRAUMAM et al., 2018).

A DPOC é uma doença sistêmica cujo manejo clínico visa a restaurar funções físicas e psicossociais, com a reabilitação pulmonar (RP) constituindo parte essencial, pois reduz sintomas como dispneia, fadiga, meIhora a capacidade de exercício e a qualidade de vida relacionada à saúde (MCCARTHY et al., 2015; YANG et al., 2019). A avaliação e o tratamento do equilíbrio são os principais focos visando à prevenção de quedas, porém poucos programas empregam uma avaliação padronizada do equilíbrio postural (BEAUCHAMP et al., 2013; MARQUES et al., 2015), embora esta tenha sido recomendada pelas Sociedades Americana e Europeia em suas diretrizes (ATS/ERS, 2013). Neste sentido, revisão crítica demonstrou que o uso de um teste breve de equilíbrio, como o timed up and go (TUG), fornece informações sobre o risco de queda em idosos e em pessoas com DPOC. Já testes longos e abrangentes, como a Escala de Equilíbrio de Berg (EEB) e o Balance Evaluation Systems Test (BESTest) permitem avaliar detalhadamente o equilíbrio visando a orientar a prescrição de exercícios (BEAUCHAMP, 2019).

Em relação ao treinamento do equilíbrio, os programas de RP são direcionados ao treinamento de músculos periféricos por meio de exercício aeróbico e de resistência e não incluem rotineiramente um componente específico de estratégias com treinamento de equilíbrio (MKACHER et al., 2015). O exercício físico pode propiciar melhora no equilíbrio e redução do risco de queda, entretanto a diretriz para prevenção de quedas preconiza o treinamento específico de equilíbrio (VERFASSER et al., 2001). Neste contexto, revisão sistemática avaliou o impacto da RP na sobrevida e risco de queda, incluindo o equilíbrio, em pacientes com DPOC estável. Os achados, entretanto, demonstraram evidências inconclusivas e limitadas dos efeitos benéficos da RP na melhora do equilíbrio ou da sobrevida. Ressalta-se que a referida revisão utilizou como grupo-controle sujeitos com DPOC que não realizaram RP e não considerou especificamente o treinamento de equilíbrio nestes programas (HAKAMY; BOLTON; MCKEEVER, 2017).

Diante da importância do equilíbrio postural como um fator de risco modificável para as taxas de quedas, torna-se necessária a realização de revisões sistemáticas especificamente direcionadas às intervenções que possam atuar sobre este desfecho, como o treinamento de equilíbrio na RP em pacientes com DPOC, visando a fornecer uma perspectiva mais precisa e evidências atuais. Diante do exposto, o objetivo deste estudo foi revisar sistematicamente a literatura sobre a eficácia da adição do treino de equilíbrio em programas de RP comparado à RP convencional na meIhora do equilíbrio postural de indivíduos com DPOC.

\section{METODOLOGIA}

A presente revisão sistemática foi realizada seguindo as diretrizes do Preferred Reporting Items for Systematic Reviews and Meta-Analyses (Prisma) 
(2009). O protocolo foi registrado no International Prospective Register of Systematic Review (Prospero) sob a identificação CRD42019128731.

\section{Estratégia de pesquisa e critérios de seleção}

Uma pesquisa bibliográfica abrangente que avaliou a eficácia do treinamento de equilíbrio em programas de RP na melhora do equilíbrio postural de indivíduos com DPOC foi realizada por dois avaliadores independentes previamente treinados em revisão sistemática, nas seguintes bases de dados eletrônicas: PubMed (MEDLINE), SciELO, LILACS, PEDro, Scopus e Web of Science, incluindo artigos publicados até junho de 2019. Para contemplar todos os artigos relevantes, também foram examinadas as listas de referências dos artigos incluídos e as revisões relacionadas. A pesquisa foi realizada sem limite quanto ao ano de publicação ou idioma. A estratégia de busca usada no PubMed é apresentada a seguir:

/(("Pulmonary Disease, Chronic Obstructive" [MeSH] OR "COPD" OR "Chronic Obstructive Pulmonary Disease" OR "COAD" OR "Chronic Obstructive Airway Disease" OR "Chronic Obstructive Lung Disease" OR "Airflow Obstruction, Chronic" OR "Airflow Obstructions, Chronic" OR "Chronic Airflow Obstructions" OR "Chronic Airflow Obstruction")) AND ("Postural Balance"[MeSH] OR "Balance, Postural" OR "Musculoskeletal Equilibrium" OR "Equilibrium, Musculoskeletal" OR "Postural Equilibrium" OR "Equilibrium, Postural" OR "postural control" OR "Postural Instability" OR "postural stability" OR "postural steadiness" OR "Postural performance" OR "balance training" OR "functional balance" OR "Balance-specific training")) AND ("Rehabilitation" [MeSH] OR "Habilitation" OR "pulmonary rehabilitation" OR "Accidental Falls"[MeSH] OR "Falls" OR "Falling" OR "Falls, Accidental" OR "Accidental Fall" OR "Fall, Accidental" OR "Slip and Fall" OR "Fall and Slip"). O MeSH "Accidental Falls" foi incluído com o objetivo de ampliar a estratégia de busca. Para os demais bancos de dados uma estratégia de pesquisa sensível foi adaptada.

\section{Critérios de elegibilidade}

Os estudos potencialmente relevantes foram incluídos após a verificação do título e dos resumos pelos dois avaliadores de forma independente com base nos seguintes critérios de inclusão:

- Sujeitos: Adultos com DPOC ( $\geq 18$ anos)

- Intervenção: Treinamento de equilíbrio em programas de RP
- Comparadores/controle: Programa de RP convencional

- Resultados: O desfecho primário foi o equilíbrio postural

Quaisquer discrepâncias entre os dois avaliadores foram sanadas com o auxílio de um terceiro revisor.

A decisão final sobre a inclusão do artigo na revisão teve como base a leitura completa dos estudos potencialmente relevantes. Foram considerados os seguintes critérios de exclusão: programas de RP sem treinamento de equilíbrio, estudos cujo desenho metodológico não era ensaio clínico, avaliação de resultados psicossociais e estudos duplicados (repetidos em bancos de dados). O nível de concordância na elegibilidade e o risco de viés dos estudos foram testados usando a estatística Kappa ( $\mathrm{K} \geq 0,65$ foi considerado para indicar concordância adequada).

\section{Extração de dados}

Os dois avaliadores, de forma independente, realizaram a extração de dados dos estudos considerados elegíveis por meio de um formulário padronizado. Para cada estudo os seguintes dados foram sistematicamente extraídos: detalhes da publicação (ano, autores e país), desfechos dos estudos, caracterização da amostra (idade, sexo, Índice de Massa Corporal - IMC - volume expiratório forçado no primeiro segundo - $V E F_{1}$ relação entre $V_{E F}$ e capacidade vital forçada - CVF_VEF 1 /CVF), metodologia do estudo (instrumentos utilizados para avaliar o equilíbrio postural, descrição da intervenção de treinamento de equilíbrio na RP quanto à frequência, duração, tipos de exercício, forma de progressão e informações sobre o grupo comparação) e resultados obtidos (método de análise, principais resultados e conclusões).

\section{Avaliação do risco de viés}

Para avaliação do risco de viés dos estudos selecionados foi utilizado pelos dois avaliadores o instrumento de risco de viés da Cochrane Collaboration (2009). Cada estudo avaliado considerou os seguintes domínios-chave: (a) viés de seleção (geração de sequência aleatória e ocultação de alocação); (b) viés de performance (cegamento de participantes e profissionais); (c) viés de detecção (cegamento dos avaliadores de desfecho); (d) viés de atrito (desfechos incompletos); (e) viés de relato (relato de desfecho seletivo); e (f) outros vieses (outras fontes de viés não abordadas em outro lugar). Cada artigo ao final da avaliação foi classificado como "alto risco" ou "baixo risco" (HIG- 
GINS; GREEN, 2011). Assim foram considerados estudos com baixa qualidade (ou seja, com alto risco de viés) ou com alta qualidade (ou seja, com baixo a moderado risco de viés) diante da presença, respectivamente, de três ou mais ou três ou menos fontes reconhecíveis de viés.

\section{RESULTADOS}

A estratégia de busca identificou 98 registros potencialmente relevantes, após exclusão das duplicatas. Posteriormente à triagem de títulos e resumos, três artigos foram selecionados para leitura do texto completo. Um estudo foi excluído devido à ausência de um grupo controle. Assim sendo, dois estudos preencheram os critérios de inclusão e foram considerados nesta revisão sistemática. O nível de concordância entre os revisores para seleção dos estudos foi de $\mathrm{k}$ $=1,00$. O diagrama de fluxo resume o processo de seleção de estudos e as razões para exclusões (Figura 1).

As características principais dos estudos incluídos na presente revisão sistemática são apresentadas na Tabela 1. Ambos os estudos foram publicados em inglês, um no ano de 2015 desenvolvido na Tunísia (MKACHER et al., 2015) e o outro em 2013 realizado no Canadá (BEAUCHAMP et al., 2013). Esta revisão inclui 107 pacientes com DPOC, dos quais 56 foram alocados para receber o treino de equilíbrio em um programa de RP e os demais foram inseridos no GC (RP convencional). Apenas os pacientes com declínio autorreferido de queda nos últimos 5 anos ou uma quase queda foram incluídos nos estudos. 0 treinamento de equilíbrio proposto foi similar nos estudos, entretanto houve diferença quanto à duração da intervenção proposta (MKACHER et al., 2015; BEAUCHAMP et al., 2013).

O equilíbrio foi mensurado por meio da Escala de Equilíbrio de Berg de 14 itens em ambos os estudos (MKACHER et al., 2015; BEAUCHAMP et al., 2013). Ademais, utilizou-se o Timed Up and Go, teste de apoio unipodal, teste de Tinetti (MKACHER et al., 2015) e Balance Evaluation Systems Test (BESTest) (BEAUCHAMP et al., 2013). A Activities-specific Balance Confidence $(A B C)$ foi utilizada para avaliar o nível de confiança na realização de atividades específicas sem que houvesse perda de equilíbrio ou se tornasse instável e o teste de caminhada de seis minutos (TC6M) realizado para avaliar a tolerância ao exercício (MKACHER et al., 2015; BEAUCHAMP et al., 2013). Além disso, diferentes desfechos foram investigados, como função

Figura 1 - Diagrama de fluxo
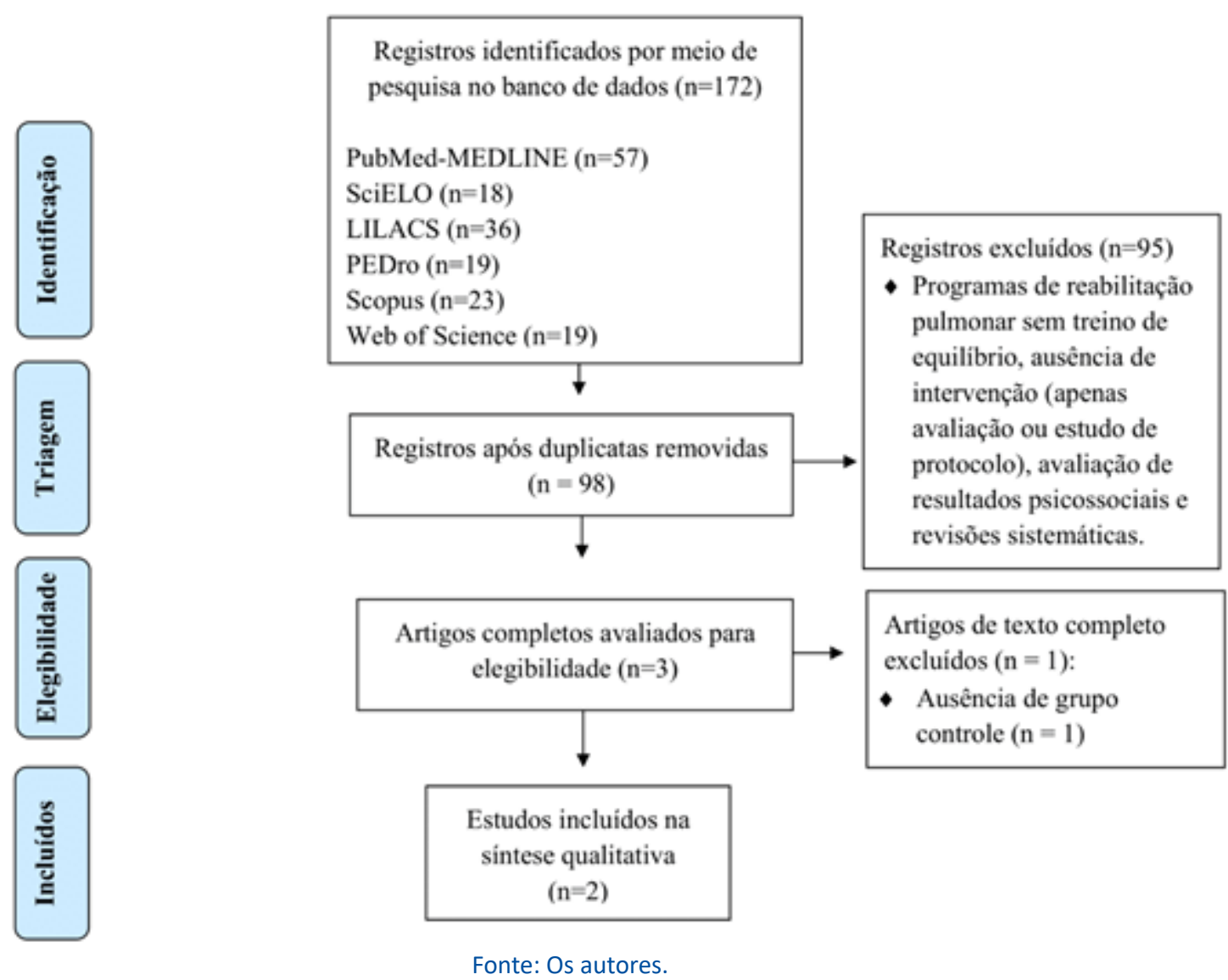
física autorreferida por meio do 36-Item Short-Form Health Survey (SF-36) e força de membros inferiores mediante o sit-to-stand de 30 segundos (STS de [30seg]) (BEAUCHAMP et al., 2013).

Em ambos os estudos o Gl submetido ao treinamento de equilíbrio e à RP convencional apresentou melhora no equilíbrio em relação ao GC submetido unicamente à RP (MKACHER et al., 2015; BEAUCHAMP et al., 2013). O treinamento de equilíbrio, no entanto, propiciou aumento significativo no nível de confiança, avaliado mediante as pontuações obtidas na escala $A B C$, durante a realização de atividades específicas em apenas um dos estudos (MKACHER et al., 2015). Houve melhora na função física autorreferida e na força muscular dos membros inferiores após o treinamento de equilíbrio (BEAUCHAMP et al., 2013). A tolerância ao exercício não apresentou diferença significativa entre os grupos após a intervenção (MKACHER et al., 2015; BEAUCHAMP et al., 2013). Os resultados estão descritos na Tabela 2, de acordo com os parâmetros considerados na análise.

Estudo conduzido por Mkacher et al. (2015) observou que no GI $100 \%$ ( $n=35)$ dos pacientes referiram perceber seu equilíbrio como "muito melhor" após o treinamento. Já no GC, 36,4\% ( $n=12)$ dos pacientes relataram ter percebido seu equilíbrio como "muito meIhor", enquanto $63,6 \%(n=21)$ não relataram nenhuma mudança (MKACHER et al., 2015). No estudo de Beauchamp et al. (2013), para 89,5\% ( $n=17)$ dos pacientes do GI o equilíbrio foi referido como "muito melhor" e para $10,5 \%(n=2)$ como "um pouco melhor". No GC, apenas $11,8 \%(n=2)$ dos pacientes relataram seu equilíbrio como "muito melhor", 41,2\% ( $n=7)$ como "um pouco melhor" e $47,1 \%(n=8)$ não relataram mudança (BEAUCHAMP et al., 2013).

Os dois estudos incluídos nesta revisão sistemática apresentaram baixo risco de viés, ou seja, alta qualidade (BEAUCHAMP et al., 2013; MKACHER et al., 2015) $(k=1,00)$. Os resultados estão descritos na Tabela 3, de acordo com os parâmetros considerados na análise.

\section{DISCUSSÃO}

A presente revisão sistemática é a primeira desenvolvida visando a avaliar a eficácia da adição do treino de equilíbrio em programas de RP comparado à RP convencional no equilíbrio postural em indivíduos com DPOC. Os resultados dos dois ensaios randomizados (MKACHER et al., 2015; BEAUCHAMP et al., 2013) incluídos nesta revisão sugerem que a adição do treino de equilíbrio em um programa de RP é eficaz na melhora do equilíbrio postural em sujeitos com DPOC.

Estudo conduzido com mais de 16 mil idosos canadenses demonstrou que a presença de DPOC foi a única condição crônica, das 13 examinadas, considerada um preditor significativo de quedas, cujos possíveis fatores contribuintes compreendem a disfunção muscular esquelética e a hipoxemia cerebral (SIBLEY et al., 2014). Beauchamp (2019) em uma recente revisão na qual demonstrou que, nos últimos dez anos, um crescente número de estudos vem apontando que o déficit no equilíbrio em sujeitos com DPOC não pode ser explicado apenas por fatores relacionados à idade. Considerando a tríade do equilíbrio postural (visão, aparelho vestibular e propriocepção), estudos têm demonstrado a ausência de associação entre déficits visuais e quedas (ROING et al., 2011), bem como as alterações nos sistemas auditivo e vestibular não são observadas com frequência em pacientes com DPOC (EL-KADY et al., 2006). As alterações proprioceptivas, no entanto, as quais contemplam aumento da dependência dos sinais proprioceptivos dos músculos do tornozelo e diminuição da dependência dos sinais proprioceptivos da coluna têm sido sugeridas como o fator de risco mais importante para o déficit de equilíbrio nesses sujeitos, especialmente naqueles com fraqueza muscular inspiratória. A inadequada orientação do tornozelo pode ser explicada por uma postura prejudicada dos músculos inspiratórios para a estabilidade do tronco, resultando em um potencial fator de risco para a incidência de quedas nesse segmento da população (JANSSENS et al., 2013).

Nesse contexto, o exercício com treinamento específico de equilíbrio tem se mostrado a única intervenção efetiva para reduzir com sucesso a taxa de risco de queda (BEAUCHAMP et al., 2016). Nesta revisão, em ambos os estudos, o treino de equilíbrio foi constituído de quatro principais exercícios que contemplaram postura, transição, marcha e fortalecimento funcional. O tempo de duração, entretanto, diferiu: em um deles perdurou por 24 semanas, totalizando 72 sessões (MKACHER et al. 2015) e no outro por 6 semanas, totalizando 18 sessões (BEAUCHAMP et al., 2013).

Considerando o desfecho equilíbrio postural, em ambos os estudos incluídos nesta revisão sistemática (MKACHER et al., 2015; BEAUCHAMP et al., 2013), a adição do treino de equilíbrio em um programa de RP foi capaz de propiciar incremento significativo na pontuação final da Escala de Equilíbrio de Berg, bem como uma diferença clinicamente importante (mu- 


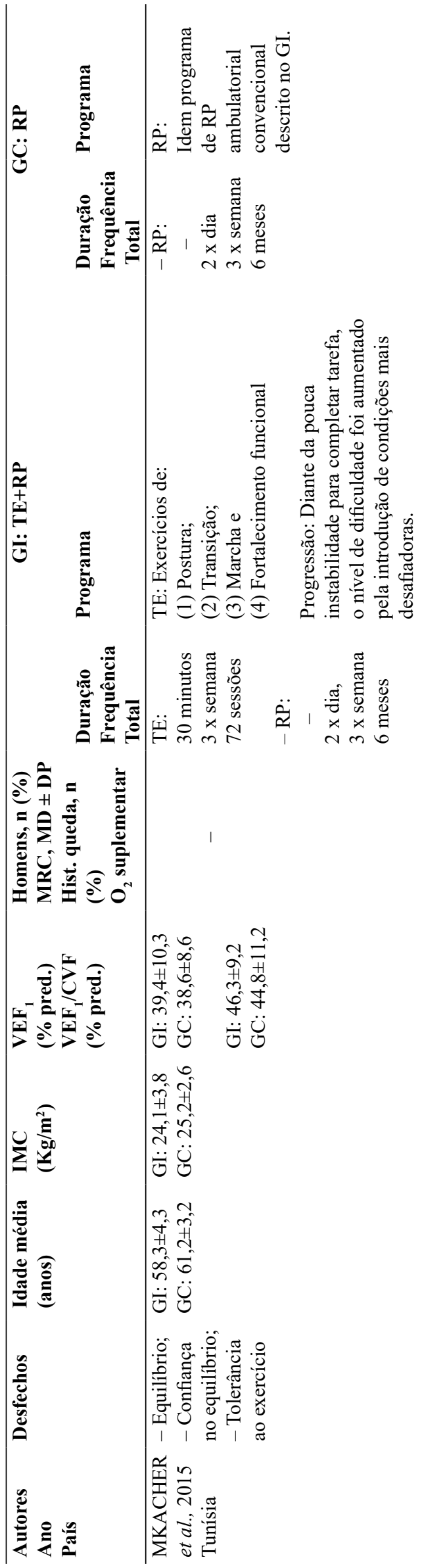

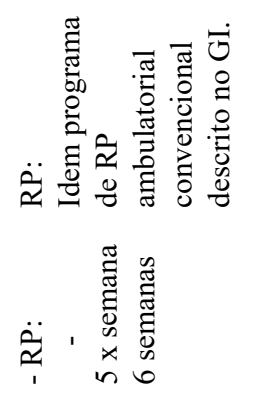
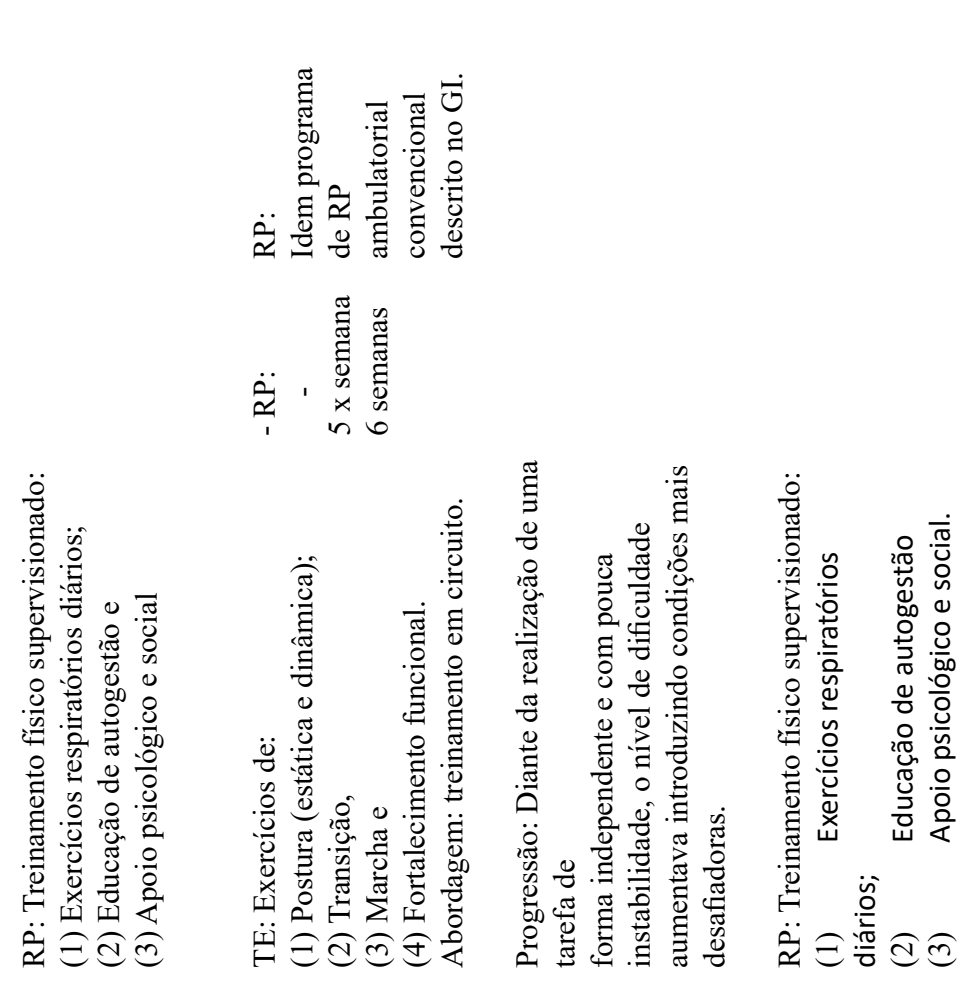


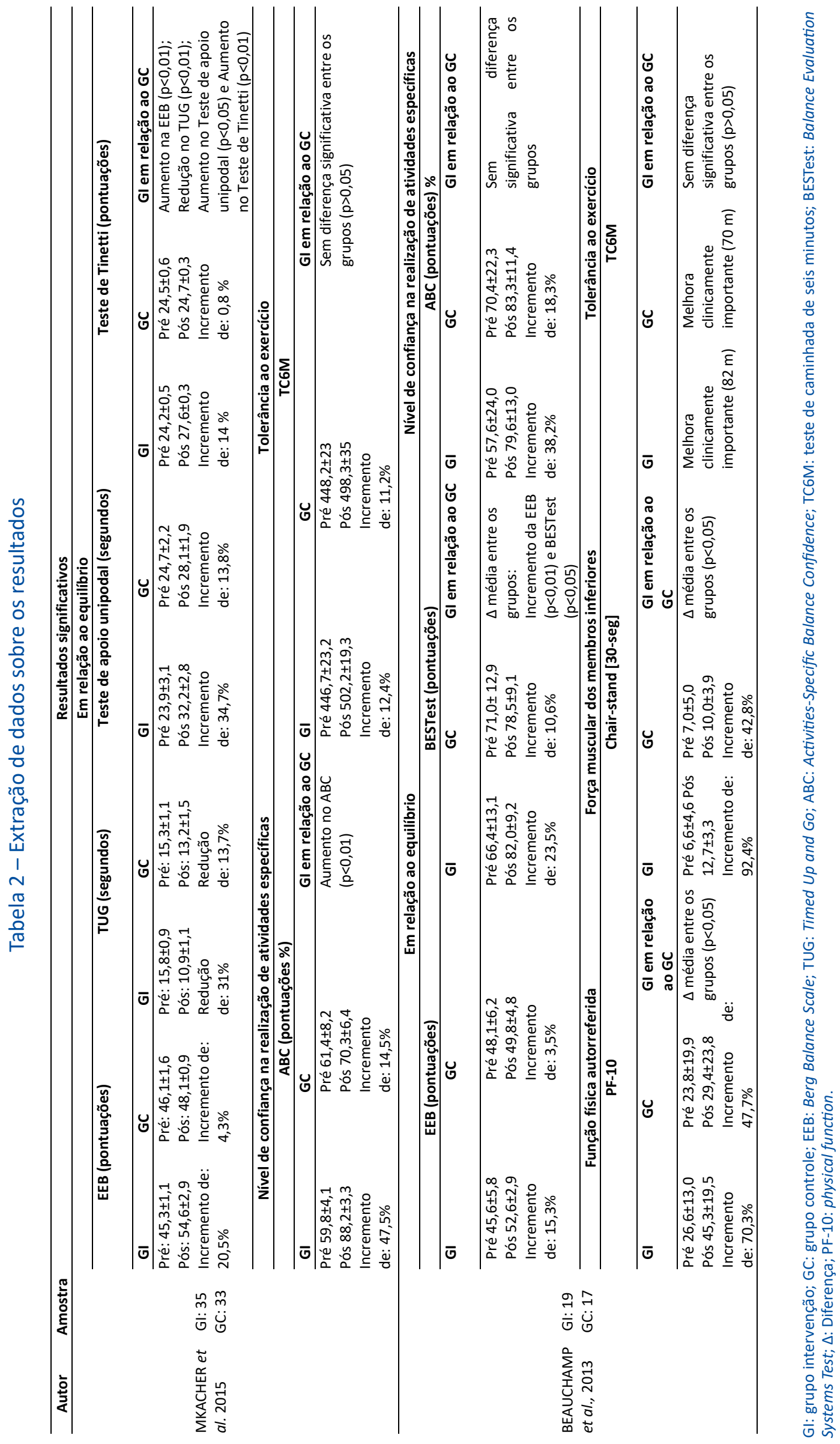


Tabela 3 - Avaliação do risco de viés dos estudos incluídos

\begin{tabular}{|c|c|c|c|c|c|c|c|}
\hline \multirow[b]{2}{*}{ Estudos } & \multicolumn{2}{|c|}{ Viés de seleção } & \multirow{2}{*}{$\begin{array}{c}\text { Viés de } \\
\text { performance } \\
\text { Cegamento de } \\
\text { participantes e } \\
\text { profissionais }\end{array}$} & \multirow{2}{*}{$\begin{array}{l}\text { Viés de detecção } \\
\text { Cegamento de } \\
\text { avaliadores de } \\
\text { desfecho }\end{array}$} & \multirow{2}{*}{$\begin{array}{c}\text { Viés de } \\
\text { atrito } \\
\text { Desfechos } \\
\text { incompletos }\end{array}$} & \multirow{2}{*}{$\begin{array}{l}\text { Viés de relato } \\
\text { Relato de } \\
\text { desfecho } \\
\text { seletivo }\end{array}$} & \multirow{2}{*}{$\begin{array}{c}\text { Outros } \\
\text { vieses } \\
\text { Outras } \\
\text { fontes de } \\
\text { viés }\end{array}$} \\
\hline & $\begin{array}{c}\text { Geração de } \\
\text { sequência aleatória }\end{array}$ & $\begin{array}{l}\text { Ocultação de } \\
\text { alocação }\end{array}$ & & & & & \\
\hline $\begin{array}{l}\text { MKACHER } \text { et } \\
\text { al. } 2015\end{array}$ & Baixo & Baixo & Incerto & Incerto & Incerto & Baixo & Baixo \\
\hline $\begin{array}{l}\text { BEAUCHAMP } \\
\text { et al. } 2013\end{array}$ & Baixo & Baixo & Baixo & Baixo & Baixo & Baixo & Baixo \\
\hline
\end{tabular}

dança de 5 a 7 pontos) em sujeitos com DPOC. Cabe destacar que essa pontuação mostra uma diferença clínica importante, pois é considerada perceptível pelo pacientes e além do erro de medição (BEAUCHAMP et al., 2016). Estudo conduzido por Mkacher et al. (2015) demonstrou redução significativa e mudança clinicamente importante no tempo de execução do Timed up and go (TUG) (0,9 - 1,4 segundos) (MESQUITA et al., 2016), aumento do tempo durante o teste de apoio unipodal e aumento significativo na pontuação total do teste de Tinetti após o treino de equilíbrio. Em virtude da ausência de um ponto de corte que defina a mudança clinicamente importante em sujeitos com DPOC para o teste de apoio unipodal e de Tinetti, ressalta-se que, em ambas as avaliações, a alteração mínima detectável, definida como a quantidade mínima de modificação que não é atribuível a um erro de medição foi alcançada (MKACHER; TABKA; TRABELSI, 2017). Beauchamp et al. (2013), contudo, demonstraram ao utilizarem o BESTest que a adição do treino de equilíbrio na RP resultou em aumento significativo e diferença clinicamente importante $(10,2$ a 17,4 pontos) (BEAUCHAMP et al., 2016) na pontuação total do referido instrumento.

Sobre o nível de confiança na realização de atividades específicas, mensurado pela escala Activities-Specific Balance Confidence (ABC), observou-se aumento deste desfecho e diferença clinicamente importante (pontuação com mudança superior a 8\%) (MKACHER; TRABKA; TRABELSI, 2017) em ambos os estudos (MKACHER et al., 2015; BEAUCHAMP et al., 2013). Em apenas um deles (MKACHER et al., 2015), entretanto, a adição do treino de equilíbrio à RP foi capaz de propiciar diferença significativa em relação à RP convencional. Possíveis explicações para este achado divergente podem estar relacionadas às diferenças no tempo de duração da intervenção proposta e na idade dos sujeitos com DPOC incluídos nos estudos, tendo em vista que no estudo de Mkacher et al. (2015) a duração foi de 24 semanas e a média da idade foi de $58,3 \pm 4,3$ anos (GI) e $61,2 \pm 3,2$ anos (GC) e no estudo de Beauchamp et al. (2013) a duração foi de 6 semanas e a média de idade de 71,9 $\pm 4,9$ anos (GI) e 67,1 $\pm 9,4$ anos (GC). Assim, sugere-se que um tempo maior de intervenção é necessário para um nível de confiança mais robusto visando à realização de atividades específicas, bem como o fato de os sujeitos serem idosos ou não pode ter influenciado neste desfecho.

Pacientes com DPOC frequentemente apresentam redução da tolerância ao exercício decorrente do sedentarismo e disfunção muscular esquelética, especialmente das fibras de contração lenta, podendo culminar em déficit no equilíbrio (JAITOVICH; BARREIRO, 2018). Neste sentido, estudos têm demonstrado que prejuízos no equilíbrio resultam em uma importante limitação funcional física em indivíduos com DPOC (BEAUCHAMP et al., 2012), contribuindo para a redução da tolerância ao exercício que é considerada um forte preditor para todas as causas de mortalidade (PUHAN et al., 2013). Considerando os achados desta revisão, os dois estudos incluídos (MKACHER et al., 2015; BEAUCHAMP et al., 2013) revelaram que ambos os grupos (GI: $56 \mathrm{~m}$ e GC: $50 \mathrm{~m}$, MKACHER et al., 2015; GI: 82 m e GC: 70 m, BEAUCHAMP et al., 2013) apresentaram uma diferença na percepção da capacidade de locomoção no TC6M (REDELMEIER et al., 1997). A adição do treino de equilíbrio, contudo, não foi capaz de promover mudanças nesta percepção. Cabe ressaltar que o estudo conduzido por Beauchamp et al. (2013) demonstrou que a adição do treino de equilíbrio à RP, de curta duração, promoveu aumento significativo na função física autorreferida e na força muscular dos membros inferiores. A justificativa para tal achado pode fundamentar-se na questão de que déficits no equilíbrio têm sido associados a níveis reduzidos de atividade física e fraqueza muscular esquelética em pacientes com DPOC (BEAUCHAMP et al., 2012).

A qualidade dos estudos incluídos nesta revisão foi avaliada por sete critérios baseados em domínios e a maioria dos itens considerados $(78,57 \%)$ foram clas- 
sificados como baixo risco de viés, incluindo parâmetros como geração de sequência aleatória, ocultação de alocação, relato de desfecho seletivo e outras formas de viés em ambos os estudos (MKACHER et al., 2015; BEAUCHAMP et al., 2013). Em um dos estudos também foi observado baixo risco de viés para os domínios: cegamento de participantes e profissionais, cegamento de avaliadores de desfecho e desfechos incompletos (BEAUCHAMP et al., 2013). Já o outro estudo, para os mesmos domínios citados anteriormente, apresentou risco de viés incerto (MKACHER et al., 2015).

Esta foi a primeira revisão sistemática que avaliou a eficácia da adição do treino de equilíbrio em programas de RP comparado à RP convencional no equilíbrio postural em indivíduos com DPOC, na qual uma busca abrangente e sistemática da literatura foi realizada, sem restrições quanto à data ou idioma de publicação, com o uso de critérios de elegibilidade explícitos e reprodutíveis, realizada de forma independente por dois avaliadores. Certas limitações, no entanto, precisam ser consideradas ao interpretar os resultados deste estudo. Primeiro, o reduzido número de artigos nos quais foram encontrados e incluídos, o que impossibilitou a realização de uma metanálise. Segundo, o tamanho amostral dos estudos incluídos pode ser considerado pequeno, entretanto sabe-se que o número de sujeitos com DPOC que participam de programa de RP ainda é extremamente baixo (SAHIN; NAZ, 2018). Terceiro, o uso de diferentes instrumentos para avaliação do equilíbrio postural muitas vezes dificulta as comparações entre os estudos.

Como sugestão de estudos futuros ressalta-se a necessidade de ensaios clínicos randomizados controlados que comparem a eficácia da adição do treino de equilíbrio à RP com a RP convencional em pacientes com DPOC, cuja metodologia utilizada seja padronizada para avaliar a intervenção proposta, confirmando ou rejeitando a tendência de superioridade dessa.

\section{CONCLUSÃO}

Os achados da presente revisão sistemática sugerem que adicionar o treino de equilíbrio em um programa de RP convencional é capaz de propiciar benefícios adicionais na melhora do equilíbrio postural em pacientes com DPOC, no entanto, em virtude do reduzido número de estudos disponíveis, não há evidência robusta para tomada de decisão clínica.

Tendo em vista as implicações negativas na saúde e o elevado ônus econômico provocado pelas quedas, a prevenção torna-se uma prioridade em serviços de saúde. Assim, espera-se a partir desta presente revisão sistemática ampliar o conhecimento sobre o treinamento de equilíbrio em programas de RP especificamente em sujeitos com DPOC.

\section{REFERÊNCIAS}

BEAUCHAMP, M. K. Balance assessment in people with COPD: An evidence-based guide. Chronic Respiratoey Disease, v. 16, n. 0, p. 1-8, Jan. 2019.

BEAUCHAMP, M. K. et al. Interpretability of Change scores in measures of balance in people With COPD. Chest, v. 149, n. 3, p. 696-703, Mar. 2016.

BEAUCHAMP, M. K. et al. A randomized controlled trial of balance training during pulmonary rehabilitation for individuals with COPD. Chest., v. 144, n. 6, p. 1.803-1.810, Dec. 2013.

BEAUCHAMP, M. K. et al. Impairments in systems underlying control of balance in COPD. Chest, v. 141, n. 6, p. 1.496-1.503, jun. 2012.

EL-KADY, M. A. et al. Study of auditory function in patients with chronic obstructive pulmonary diseases. Hearing Research, v. 212, n. 1, p. 109-116, Feb. 2006.

GOLD. Global Initiative for Chronic Obstructive Lung Disease. Global Strategy for the Diagnosis, Management and Prevention of COPD. 2019. Disponível em: https://goldcopd. org/wp-content/uploads/2018/11/GOLD-2019-POCKET-GUIDE-FINAL_WMS.pdf. Acesso em: 21 jul. 2019.

GRAAT-VERBOOM, L. et al. Osteoporosis in COPD outpatients based on bone mineral density and vertebral fractures. Journal of Bone and Mineral Research, v. 26, n. 3, p. 561-568, Mar. 2011.

GRAUMAM, R. Q. et al. Increased rate of osteoporosis, low lean mass, and fragility fractures in COPD patients: association with disease severity. Osteoporosis International, v. 29, n. 6, p. 1.457-1.468, June 2018.

HAKAMY, A.; BOLTON, C. E.; MCKEEVER, T. M. The effect of pulmonary rehabilitation on mortality, balance, and risk of fall in stable patients with chronic obstructive pulmonary disease: A systematic review. Chronic Respiratory Disease, v. 14, n. 1, p. 54-62, 2017.

HIGGINS, J. P.; GREEN, S. Cochrane handbook for systematic reviews of interventions. version 5.1.0. Oxford, UK: The Cochrane Collaboration, 2011. Disponível em: www.cochrane-handbook.org. Acesso em: 21 jul. 2019.

JAITOVICH, A.; BARREIRO, E. Skeletal Muscle Dysfunction in Chronic Obstructive Pulmonary Disease What We Know and Can Do for Our Patient. American Journal of Respiratory and Critical Care Medicine, v. 198, n. 2, p. 175-186, 2018.

JANSSENS, L. et al. Proprioceptive changes impair balance control in individuals with chronic obstructive pulmonary disease. PLOS ONE, v. 8, n. 3, p. e57949, 2013. 
KOKTURK, N. et al. Vitamin D deficiency: what does it mean for chronic obstructive pulmonary disease (COPD)? A comprehensive review for pulmonologists. Clinical Respiratory Journal, v. 12, n. 2, p. 382-397, Feb. 2018.

LIU, W. T. et al. Low bone mineral density in COPD patients with osteoporosis is related to low daily physical activity and high COPD assessment test scores. International Journal of COPD, v. 10, n. 1, p. 1.737-1.744, Sept. 2015.

MARQUES, A. et al. Effects of a pulmonary rehabilitation program with balance training on patients with COPD. Journal of Cardiopulmonary Rehabilitation and Prevention, v. 35, n. 2, p. 154-8, Mar./Apr. 2015.

MCCARTHY, B. et al. Pulmonary rehabilitation for chronic obstructive pulmonary disease. Cochrane Database Systematic Reviews, v. 23, n. 2, p. 1-67, Feb. 2015.

MESQUITA, R. et al.; Measurement properties of the Timed Up \& Go test in patients with COPD. Chronic Respiratory Disease, v. 13, n. 4, p. 344-352, July 2016.

MKACHER, W. et al. Effect of 6 months of balance training during pulmonary rehabilitation in patients with COPD. Journal Cardiopulmonary Rehabilitation and Prevention, $v$. 35, n. 3, p. 207-213, May./June 2015.

MKACHER, W.; TABKA, Z.; TRABELSI, Y. Minimal detectable change for balance measurements in patients with COPD. Journal of Cardiopulmonary Rehabilitation and Prevention, v. 37, n. 3, p. 223-228, May. 2017.

MOHER, D. et al. Preferred reporting items for systematic review and meta-analysis protocols (PRISMA-P) 2015 statement. Systematic Reviews, v. 4, n. 1, p. 1-9, Jan. 2015.

MOON, Ji-Yong.; SIN, D. D. Inhaled corticosteroids and fractures in chronic obstructive pulmonary disease: current understanding and recommendations. Current Opinion in Pulmonary Medicine, v. 25, n. 2, p. 165-172, Mar. 2019.

PORTO, A. et al. Risk of fall in patients with COPD. Thorax, v. 73, n. 11, p. 1.079-1.080, Nov. 2018.

PORTO, E. F. et al. Comparative postural control in COPD patients and healthy individuals during dynamic and static activities. Journal of Cardiopulmonary Rehabilitation and Prevention, v. 37, n. 2, p. 139-145, Mar. 2017.

PUHAN, M. A. et al. Simple functional performance tests and mortality in COPD. European Respiratory Journal, v. 42, n. 4, p. 956-963, Oct. 2013.

RAMON, M. A. et al. The dyspnea-inactivity vicious circle in COPD: development and external validation of a conceptual model. European Respiratory Journal, v. 52, p. 1-11, July 2018.

REDELMEIER, D. A. et al. Interpreting small differences in functional status: the Six Minute Walk test in chronic lung disease patients. American Journal of Respiratory and Critical Care Medicine, v. 155, n. 4, p. 1.278-1.282, 1997.

ROCHA, F. R. et al. Relação da mobilidade diafragmática com função pulmonar, força muscular respiratória, dispneia e atividade física de vida diária em pacientes com DPOC. Jornal Brasileiro de Pneumologia, v. 43, n. 1, p. 32-37, jan./ fev. 2017.
ROING, M. et al. Falls in people with chronic obstructive pulmonary disease: an observational cohort study. Respiratory Medicine, v. 105, n. 3, p. 461-469, Mar. 2011.

SAHIN, H.; NAZ, I. Why are COPD patients unable to complete the outpatient pulmonary rehabilitation program? Chronic Respiratory Disease, v. 15, n. 4, p. 411-418, Apr. 2018.

SIBLEY, K. M. et al. Chronic disease and falls in community-dwelling Canadians over 65 years old: a population-based study exploring associations with number and pattern of chronic conditions. BMC Geriatrics, v. 14 , n. 22 , p. 1-11, 2014.

SPRUIT, M. A. et al. An official American Thoracic Society/ European Respiratory Society statement: key concepts and advances in pulmonary rehabilitation. American Journal of Respiratory and Critical Care Medicine, v. 188, n. 8, p. 1364, Oct. 2013.

SHUMWAY-COOK, A.; WOOLLACOTT, M. Motor Control Translating Research into Clinical Practice. 3rd ed. Philadelphia: Lippincott Williams and Wilkins, 2007.

VERFASSER, U. et al. Guideline for the prevention of falls in older persons. American Geriatrics Society, British Geriatrics Society, and American academy of orthopaedic surgeons panel on falls prevention. Journal of the American Geriatrics Society, v. 49, n. 5, p. 664-72, May. 2001.

WHO. World Health Organization. The top 10 causes of death. 2018. Disponível em: https://www.who.int/news-room/fact-sheets/detail/the-top-10-causes-of-death. Acesso em: 9 maio 2020.

YANG, J. et al. Significance of pulmonary rehabilitation in improving quality of life for subjects with COPD. Respiratory Care, v. 64, n. 1, p. 99-107, Jan. 2019. 